\title{
COMMENTARY
}

\section{Probing with the ventilator}

\author{
Martin J Tobin \\ See related research by Barwing et al., http://ccforum.com/content/17/4/R182
}

\begin{abstract}
Neurally adjusted ventilatory assist operates through recordings of electrical activity of the diaphragm (EAdi). Barwing and colleagues found increases in EAdi in weaning-failure patients, although the values were not significantly different from weaning-success patients. Future studies will need to carefully control for the considerable biological noise evident in EAdi recordings.
\end{abstract}

Although a mechanical ventilator is primarily a life-saving device, its recordings of pressure and flow offer a means for undertaking detailed assessment of respiratory function. Over the course of a few seconds, it is possible to obtain a more complete assessment of lung and chest-wall mechanics than can be achieved after an hour's assessment of an ambulatory patient in a pulmonary-function laboratory. In both settings, evaluation of the respiratory centers is typically omitted. This omission is partly because assessment involves use of a distinct set of techniques, such as $\mathrm{CO}_{2}$ rebreathing, but the major reason is cognitive: interpretation of the results is highly complex and difficult to incorporate into routine clinical decision-making. The introduction of a new ventilator mode makes it possible for intensivists to outperform the diagnostic capabilities of pulmonologists one more time.

In the previous issue of Critical Care, Barwing and colleagues [1] evaluate the usefulness of neurally adjusted ventilatory assist (NAVA), which requires esophageal electrodes to record electrical activity of the diaphragm (EAdi), in patients being considered for a trial of weaning from mechanical ventilation. That the Göttingen investigators should turn to this group of patients is not surprising. Weaning failure offers one of the supreme experiments of nature. Investigators can select

Correspondence: mtobin2@lumc.edu

Division of Pulmonary and Critical Care Medicine, Edward Hines Jr Veterans Affairs Hospital and Loyola University of Chicago Stritch School of Medicine, Hines, IL 60141, USA a time when they are able to assemble their research armamentarium at a patient's bedside and observe the natural history of acute respiratory failure unfold over the course of a half-hour or so [2]. If a struggling patient is not reconnected to the ventilator, he or she will die. Few experiments in medicine come closer to life-or-death circumstances.

In this feasibility study, Barwing and colleagues observed increases in respiratory motor output in weaning-failure patients. Although the values of EAdi were numerically higher in weaning-failure than in weaning-success patients, the difference between the groups did not attain statistical significance - in large part because EAdi displayed considerable variability on a breath-to-breath basis (see Figure three of their paper), and also because of considerable overlap in the individual values in the two groups (evident in Figure one of their paper).

In 1986, my colleagues and I [3] published a study on control of breathing in patients undergoing weaning trials. We recorded mean inspiratory flow $\left(\mathrm{V}_{\mathrm{T}} / \mathrm{T}_{\mathrm{I}}\right)$ as a measure of respiratory motor output. Contrasted with EAdi, which is recorded with electrodes lying immediately adjacent to the source of respiratory muscle contraction [4], $\mathrm{V}_{\mathrm{T}} / \mathrm{T}_{\mathrm{I}}$ provides a less direct measure of respiratory motor output because it is also influenced by changes in respiratory mechanics. Despite this technical limitation, we observed increases in $V_{T} / T_{I}$ in every patient who failed a weaning trial. This finding was surprising because conventional wisdom dictated that the development of acute hypercapnia, consequent to alveolar hypoventilation, was caused by a decrease in respiratory motor output. Instead, rapid shallow breathing with a low tidal volume, and inevitable increase in dead space-to-tidal volume ratio, was the reason for the patients' $\mathrm{CO}_{2}$ retention. Indeed, hypercapnia and activation of other sensory afferents lead to an increase in respiratory drive [5].

The reason we were successful in detecting significantly higher values of respiratory motor output in weaning-failure than weaning-success patients, in contrast to Barwing and colleagues, relates to the methods of data analysis. We collected hundreds of $\mathrm{V}_{\mathrm{T}} / \mathrm{T}_{\mathrm{I}}$ values in each 
patient, sufficient to perform frequency-histogram analysis in individual patients [3]. Through signal averaging, we were able to compensate for the marked breath-to-breath variations in respiratory motor output.

As originally shown in our 1986 study, and in hundreds of studies since then, rapid shallow breathing is the physiological hallmark of weaning failure. Based on our observations, Karl Yang and I [6] subsequently tested the accuracy of an index of rapid shallow breathing, the frequency-to-tidal volume ratio $\left(f / \mathrm{V}_{\mathrm{T}}\right)$, as a means to screen for weaning readiness. We found it more reliable than all other weaning predictors, and so it remains to this day. Barwing and colleagues did not find $f / V_{T}$ to be significantly different between their two groups of patients, but here the explanation lies in the occurrence of test-referral bias [7]. Entry into their study required patients to have an $f / V_{T}$ value of less than 105, which means the investigators were no longer in a position to test the reliability of $\mathrm{f} / \mathrm{V}_{\mathrm{T}}$ as a predictor of weaning outcome.

While we know more about weaning failure than we did in the 1980s, we still do not know the mechanism of its physiological hallmark - rapid shallow breathing - and our understanding of neuromuscular control of breathing remains superficial. Barwing and colleagues believe that the NAVA ventilator may prove useful in unraveling these mechanisms. From the raw data evident in their report, it is evident that gaining physiological insights through use of EAdi will require sophisticated approaches to time-series analysis to minimize the influence of biological noise.

\section{Abbreviations}

EAdi: Electrical activity of the diaphragm; $f N_{\mathrm{T}}$ : Frequency-to-tidal volume ratio; NAVA: Neurally adjusted ventilatory assist; $V_{T} / T_{1}$ : Mean inspiratory flow.

\section{Competing interests}

MJT declares receipt of royalties from McGraw-Hill for two books published on critical care medicine.

Published: 03 Oct 2013

\section{References}

1. Barwing C, Pedroni C, Olgemöller U, Quintel M, Moerer O: Electrical activity of the diaphragm (EAdi) as a monitoring parameter in difficult weaning from respirator: a pilot study. Crit Care 2013, 17:R182.

2. Tobin MJ, Jubran A: Weaning from mechanical ventilation. In Principles and Practice of Mechanical Ventilation. 3rd edition. Edited by Tobin MJ. New York: McGraw-Hill, Inc; 2012:1185-1220.

3. Tobin MJ, Guenther SM, Perez W, Mador MJ, Semmes BJ, Allen SJ, Lodato RF, Dantzker DR: The pattern of breathing during successful and unsuccessful trials of weaning from mechanical ventilation. Am Rev Respir Dis 1986, 134:1111-1118.

4. Sinderby C, Beck JC: Neurally adjusted ventilatory assist. In Principles and Practice of Mechanical Ventilation. 3rd edition. Edited by Tobin MJ. New York: McGraw-Hill, Inc; 2012:351-375.

5. Tobin MJ, Laghi F, Jubran A: Ventilatory failure, ventilator support and ventilator weaning. Compr Physiol 2012, 2:2871-2921.
6. Yang K, Tobin MJ: A prospective study of indexes predicting the outcome of trials of weaning from mechanical ventilation. N Engl J Med 1991 324:1445-1450.

7. Tobin MJ, Jubran A: Variable performance of weaning-predictor tests: role of Bayes' theorem and spectrum and test-referral bias. Intensive Care Med 2006, 32:2002-2012.

$10.1186 /$ cc13038

Cite this article as: Tobin: Probing with the ventilator.

Critical Care 2013, 17:198 